\title{
Effect of Traffic Uncertainities on the Design of Mixed Line Rate (MLR) Optical Networks
}

\author{
Sridhar Iyer and Shree Prakash Singh
}

\begin{abstract}
In the existing studies on Mixed Line Rate (MLR) optical networks, the network design methodology is based on the assumption of deterministic traffic, and hence, the effect of traffic uncertainty on the design of an MLR network remains an open problem of research. In this study, we upgrade our previously proposed cost-efficient mixed integer linear program (MILP) formulation for an MLR network, which considered a specific mean traffic for every network source-destination pair. Our upgraded model employs an optimization technique to account for the traffic uncertainties that an actual MLR optical network may encounter. Our simulation results show that (i) if the MLR network is cost-optimized under the assumption that approximately $10-20 \%$ of the demands are at their maximum (or peak) value then, the network demonstrates robustness to traffic peaks in approximately all the other demands, and (ii) the saturation of network cost for a number of sourcedestination pairs is network topology dependent.
\end{abstract}

Keywords-Mixed line rate, traffic, MILP, cost-efficient, optimization.

\section{INTRODUCTION}

With the emergence of increased and diversified data sharing patterns, the requirement for high speed optical networks has increased, and such future optical networks must also be able to serve the traffic comprising of heterogeneous demands. To meet the demand(s) of various services, the $10 \mathrm{Gbps}$ (i.e., legacy) transport optical networks have had to be upgraded to the 40 and/or 100Gbps networks. However, with such an upgrade simultaneously, the major challenge is to keep the network cost to a minimum value. In a mixed line rate (MLR) optical network, 10/40/100 Gbps co-exist on varied wavelengths within the same fiber, and planning such a network reduces the network cost owing to the volume discount of the high bit-rate transponders [1]. Also, MLR networks (i) relax the network design limitations by their ability to choose a specific line rate dynamically, as per each traffic demand, and (ii) use the appropriate number and line rate of the wavelengths on every link which solves asymmetry in the traffic as well as the network [2].

Existing studies have shown that under most traffic loads,

Manuscript received January 25, 2017, revised May 6, 2017

Sridhar Iyer is with Department of ECE, Jain College of Engineering, T S. Nagar Hunchanatti Cross-Machhe, Belagavi, Karnataka, India - 590014; e-mail: sridhariyer1983@gmail.com.

Shree Prakash Singh is with Division of ECE, Netaji Subhas Institute of Technology, Sector-3, Dwarka, New Delhi-110078.

e-mail: sps_nsit@yahoo.co.uk in comparison to a single-line-rate (SLR) optical network, an MLR optical network is both, cost and energy-efficient [1, 35]. Further, in MLR networks, based on the various scenarios, the overall cost incurred is different, which can be explained as follows: let there occur a $100 \mathrm{Gbps}$ demand between two nodes $a-b$ of the network. To satisfy such a demand, there may exist multiple paths which are connected via the fiber links between the two network nodes $a-b$. Also, it may occur that the demand (i) is set up using a transparent (i.e., an all-optical channel (wavelength)) resulting in minimum network cost, or (ii) at the increased load values, due to the signal reach constraint (which restricts a high bitrate signal to traverse only a short distance before the regeneration requirement), there is no end-to-end transparent route, and hence, between the multiple channels, the demand will require splitting up. Further, the used channels may traverse via the same or through different fibers, and therefore, the overall network cost incurred will be different.

In the existing studies, the design methodology is based on the assumption that the traffic is deterministic i.e., the traffic is specified by a matrix containing a-priori (i.e., forecasted) average traffic between the various sourcedestination $(s-d)$ pairs which; however, is not a realistic assumption. In reality, an actual MLR network tries to satisfy demands of the traffic with uncertainties, and in view of the aforementioned, we extend our previous study [6] wherein, a mixed integer linear program (MILP) model was formulated to investigate the launch power and placement of regenerator(s)'s effects on the MLR network design. The previously proposed model's objective was to lower the total network cost; however, the model assumed the traffic to be deterministic. In the current work, our MILP model considers the traffic between each $s-d$ pair to randomly fluctuate about a mean value with a specified variance i.e., we modify our previously developed MILP so as to capture the randomness in the traffic behaviour for which an optimization technique detailed in [7] is employed.

Rest of the article is structured as follows. Section II details the MLR network design. In section III, the simulation results are presented. Finally, section IV concludes the study.

\section{II.MLR NETWORK DESIGN}

In the MLR network design, for a given $s-d$ pair, the feasibility of a specific bit-rate is set by the lightpath(s) Bit Error Rate (BER). The BER model detailed in $[8,9]$ is used in this study which takes into consideration the following 
Physical Layer Impairments (PLIs): the Amplified Spontaneous Emission (ASE) noise, Cross-Phase Modulation (XPM), Four-Wave Mixing (FWM), and dispersion. The ASE noise, XPM and FWM generate the beat noise (i.e., crosstalk) terms at the receiver. Further, assuming that the signal power for bit ' 0 ' is zero, the BER and the noise powers are given as

$$
B E R=\frac{1}{4}\left[\operatorname{erfc}\left(\frac{I_{T}}{\sqrt{2} \sigma_{0}}\right)+\operatorname{erfc}\left(\frac{I_{S 1}-I_{T}}{\sqrt{2} \sigma_{1}}\right)\right],
$$

where

$$
I_{T}=\frac{\sigma_{0} \cdot I_{S 1}+\sigma_{1} \cdot I_{S 0}}{\sigma_{0}+\sigma_{1}}
$$

and $I_{S 1}$ and $I_{S 0}$ are the signal currents due to the reception of bit ' 1 ' and ' 0 ' respectively. Further,

$$
\begin{aligned}
\sigma^{2}(1) & =\sigma_{\text {signal }}^{2}+\sigma_{A S E-\text { shot }}^{2}+\sigma_{A S E-\text { signal }}^{2}+\sigma_{A S E-A S E}^{2} \\
& +\sigma_{A S E-F W M}^{2}+\sigma_{\text {signal-FWM }}^{2}+\sigma_{\text {signal-XPM }}^{2}+\sigma_{\text {Thermal }}^{2}
\end{aligned}
$$

and

$$
\begin{aligned}
\sigma^{2}(0)= & \sigma_{A S E-s h o t}^{2}+\sigma_{A S E-A S E}^{2}+, \\
& \sigma_{A S E-F W M}^{2}+\sigma_{\text {Thermal }}^{2}
\end{aligned}
$$

where in (3) and (4), $\sigma_{\text {Thermal }}^{2}, \sigma_{\text {shot }}^{2}, \sigma_{\text {signal }}^{2}$ are the thermal noise, the shot noise, and the signal noise variance respectively, $\sigma_{A S E-s h o t}^{2}, \sigma_{A S E-A S E}^{2}, \sigma_{A S E-F W M}^{2}$ are the ASEshot noise, ASE-ASE and ASE-FWM beat noise respectively, and $\sigma_{A S E-\text { signal }}^{2}, \sigma_{\text {signal-FWM }}^{2}, \sigma_{\text {signal-XPM }}^{2}$ are the ASE-signal, signal-FWM and signal-XPM beat noise respectively.

Further, the design steps in our work are detailed as follows:

1) Initially, the $k$-shortest paths ( $k$-SPs) between every network topology $s$ - $d$ pair are determined.

2) Then, over the possible routes, for a candidate lightpath, the BER is evaluated with a certain format of modulation; hence, it is checked whether the evaluated BER is less than the specified threshold or not.

3) The above step is repeated for all the line rates, hence, based on the BER threshold, specific line rate feasibility (or viability) for every $s$ - $d$ pair is ensured.

4) For few $s$ - $d$ pairs which are distant apart, and have nonviable higher line rates, the above procedure is repeated with the appropriate modulation format, whose extended reach makes few more lightpaths feasible over the higher line rates, which were infeasible with the previously used format owing to constraint set by the BER threshold.

5) With this pre-processed data as the input, a mathematical formulation of the problem is developed in the form of an MILP, which is detailed as follows:

\section{Input:}

$G(V, E)$ : Network topology comprising of a set of $V$ nodes and a set of $E$ links;
$T=\left[\Lambda_{s-d}\right]$ : Matrix consisting of the traffic having the total Gbps requests of $\Lambda_{s-d}$ between an $s$ - $d$ pair; $R=\left[R_{1}, R_{2}, \ldots . . R_{k}\right]:$ Line rate available set; $T P_{k}$ : Transponder (with rate $R_{k}$ ) cost;

$A$ : Cost of an amplifier;

$A_{m n}$ : On a fiber, the amplifier numbers over the link with nodes $m$ and $n$. For a span distance $L=80 \mathrm{~km}$ between adjacent amplifiers (EDFAs), the amount of EDFAs for the link of a fiber is given as $A_{m n}=\left[L_{m n} / L-1\right]+2$; where, $L_{m n}$ denotes length of span of the fiber between $m$ and $n$. $C_{p}$ : Electronic processing (per Gbps) cost i.e., cost of OEO conversion.

$l_{i j}:$ Lightpath length in $\mathrm{km}$ between an $s-d$ pair;

$l_{m n}:$ Link (physical) between $m$ and $n$;

$W$ : Maximum amount(s) of the wavelength(s) on a link; $\lambda \in\{1,2, \ldots . W\}$

$B$ : BER threshold which is set to $10^{-12}$. Any lightpath with a BER greater than the threshold is rejected;

$B E R_{i j k \lambda}$ : BER of a lightpath between an $s-d$ pair $i-j$ at a line rate $R_{k}$ and a wavelength $\lambda$;

$b_{i j k \lambda}=\left\{\begin{array}{ll}1 & \text { for } B E R_{i j k \lambda} \leq B \\ 0 & \text { else }\end{array} \quad \forall(i, j), k, \lambda ;\right.$ denotes the lightpath feasibility based on the threshold BER. Hence, $b$ determines the feasibility of a lightpath between a $i j k \lambda$

node(s) pair over a specific wavelength and bit rate.

$P_{m n}$ : Lightpath(s) set passing through the link $l_{m n}$.

\section{Variables:}

$X_{i j k \lambda}$ : Lightpath(s) number(s) at a rate $R_{k}$ and a wavelength $\lambda$ between the nodes $i$ - $j$;

$F_{i j}^{s d}$ : Between $s$ - $d$, traffic which is routed on a lightpath $i$ - $j$. $E_{j}$ : Variable (integer) which denotes the data amount that is carried by the lightpaths ending at node $j$.

\section{Problem formulation:}

Minimize the overall network cost which consists of (i) number of transponders and the fiber amplifiers, and (ii) the electronic processing used for setting up the multi-hop connections (i.e., the $s-d$ connections whose establishment occurs over many i.e., multiple lightpaths.), which is mathematically given as follows:

Objective:

$$
\begin{aligned}
\text { Minimize } & \sum_{\lambda} \sum_{i j} \sum_{k} X_{i j k \lambda} \cdot T P_{k}+\sum_{m, n} A \cdot A_{m n} \cdot F_{m n}+ \\
& \sum_{j} E_{j} \cdot C_{p}
\end{aligned}
$$

The objective function in (5) is constrained by: 
(i) the capacity constraint given as

$$
\sum_{\lambda} \sum_{k} R_{k} \cdot b_{i j k \lambda} \cdot X_{i j k \lambda} \geq \sum_{s, d} F_{i j}^{s d} \quad \forall(i, j)
$$

(ii) the constraint to avoid wavelength clash given as

$$
\sum_{i, j \in P_{m n}} \sum_{k} b_{i j k \lambda} \cdot X_{i j k \lambda} \leq F_{m n} \quad \forall(m, n), \forall \lambda,
$$

(iii) the conservation of traffic flow on each path given as

$$
\sum_{i} F_{i j}^{s d}-\sum_{i} F_{j i}^{s d}=\left(\begin{array}{ll}
\Lambda_{s d} & \text { for } s=j \\
-\Lambda_{s d} & \text { for } d=j \quad \forall(i, j) \forall(s, d) \\
0 & \text { otherwise }
\end{array}\right.
$$

(iv) the total of flows ending at node $j$ i.e., sum traffic at every node requiring electronic processing

$$
E_{j}=\sum_{s, d} \sum_{i} F_{i j}^{s d} \quad \forall i \neq s, \forall j \neq d
$$

The solution to the above formulated MILP consists of lightpath(s) set between $s-d$ pairs over the various bit-rates and wavelengths, also accommodating all the requests. In the overall solution, the obtained set of lightpaths is a subset of the previously evaluated viable lightpath(s) set, whose viability is defined by value of BER of the path being below a certain threshold or not.

Further, the need for optimization to incorporate the nondeterministic traffic can be explained as follows: let $\bar{\Lambda}_{s d}$ be the traffic mean and $\tilde{\Lambda}_{s d}$ the traffic variance above the average, at various times. Hence, for every $s$ - $d$ pair, the traffic can be shown as $\bar{\Lambda}_{s d}+\tilde{\Lambda}_{s d}$. Also, at any specific time, the probability of the total $s$ - $d$ pairs having the traffic volumes at peak values is very low. Hence, a parameter $\xi \in\{0,1,2, \ldots \ldots,|M|\}$ is defined, where $M$ is the total number of network $s$ - $d$ pairs. For $\xi=0$, the traffic is deterministic i.e., all the traffic demands remain at their mean value; however, for $\xi \neq 0$, the traffic at $\xi$ number of $s-d$ pairs is at its peak value, which is given as $\bar{\Lambda}_{s d}+\tilde{\Lambda}_{s d}$. The concept of simultaneous traffic peaks is explained in Fig. 1 where, a 3-node network is shown as an example to illustrate, for each network $s$ - $d$ pair, traffic for which 2 simultaneous peaks occur.

Hence, in order to ensure that the formulated MILP model incorporates traffic uncertainties, following modifications are made to the previous MILP model:

1. The $\Lambda_{s d}$ 's in (8) are replaced by the mean traffic $\bar{\Lambda}_{s d}$.

Hence, (8) is modified as

$$
\sum_{i} F_{i j}^{s d}-\sum_{i} F_{j i}^{s d}=\left(\begin{array}{lll}
\bar{\Lambda}_{s d} & \text { for } s=j \\
-\bar{\Lambda}_{s d} & \text { for } d=j \\
0 & \text { otherwise }
\end{array} \quad \forall(i, j) \forall(s, d)\right.
$$

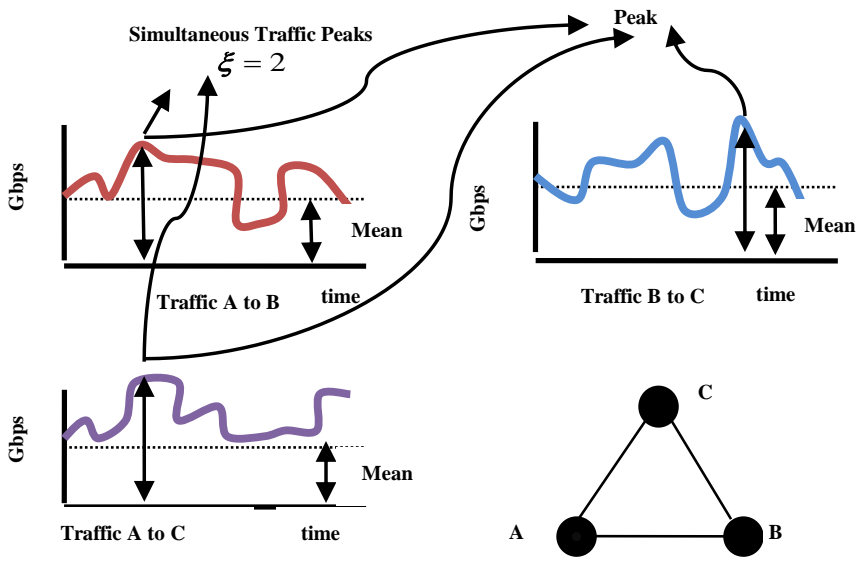

Fig.1. Characterization of the traffic and the occurrence of 2 traffic peaks at the same instant in a 3-node topology.

2. The capacity constraint given in (6) is modified as

$$
\begin{aligned}
& \sum_{\lambda} \sum_{k} R_{k} \cdot X_{i j k \lambda} \cdot b_{i j k \lambda} \geq \sum_{s, d} F_{i j}^{s d}+ \\
& \max _{L \in M,|L| \leq \xi}\left\{\sum_{s \cdot d \in L} \frac{\tilde{\Lambda}_{s d}}{\bar{\Lambda}_{s} d^{\prime}} F_{i j}^{s d}\right\} \quad \forall(i, j)
\end{aligned}
$$

where $L$ denotes a subset of the possible $M^{\prime} s$ i.e., $s$ - $d$ pairs, and further, contains all those $s$ - $d$ pairs whose traffic deviates from the mean value.

Further, by using the method of LP-duality detailed in [10], the $\max _{L \in M,|L| \leq \xi}\left\{\sum_{s^{\prime} d^{\prime} \in L} \tilde{\Lambda}_{s^{\prime} d^{\prime}} / \bar{\Lambda}_{s^{\prime} d^{\prime}}, F_{i j}^{s^{\prime} d^{\prime}}\right\}$ part of (11) can be simplified as follows:

$$
\begin{aligned}
\max _{L \in M,|L| \leq \xi}\left\{\sum_{s^{\prime} d^{\prime} \in L} \frac{\tilde{\Lambda}_{s^{\prime} d^{\prime}}}{\bar{\Lambda}_{s^{\prime} d^{\prime}}} F_{i j}^{s^{\prime} d^{\prime}}\right\} & \left.=\max \sum_{s, d} \frac{\tilde{\Lambda}_{s d}}{\bar{\Lambda}_{s d}} F_{i j}^{s d}\right\} z_{i j}^{s d} \\
& =\min \sum_{s, d} p_{i j}^{s d}+\chi_{i j} \xi
\end{aligned}
$$

s.t.: $\sum_{s, d} z_{i j}^{s d} \leq \xi \quad$ s.t.: $p_{i j}^{s d}+\chi_{i j} \geq \frac{\tilde{\Lambda}_{s d}}{\bar{\Lambda}_{s d}} F_{i j}^{s d}$,

where $z_{i j}^{s d}$ 's are the binary variables denoting whether a $s-d$ pair is a part of the subset $L$ or not, and the dual variables $\chi_{i j}$ 's and $p_{i j}^{s d}$ 's denote the constraints $\sum_{s, d} z_{i j}^{s d} \leq \xi$ and $z_{i j}^{s d} \leq 1$ respectively. Using the aforementioned derivations, two new constraints are obtained which replace (6) from the previous MILP formulation. The two new constraints are given as

$\sum_{\lambda} \sum_{k} R_{k} \cdot X_{i j k \lambda} \cdot b_{i j k \lambda} \geq \sum_{s, d} F_{i j}^{s d}+\sum_{s, d} p_{i j}^{s d}+\chi_{i j} \xi \quad \forall(i, j)$ 


$$
-\chi_{i j}+\frac{\tilde{\Lambda}_{s d}}{\bar{\Lambda}_{s d}} F_{i j}^{s d}-p_{i j}^{s d} \leq 0 \quad \forall i, j, \forall s, d
$$

For details on the derivations of (14) and (15), the reader is referred to $[7,10]$. Finally, the modified MILP formulation that incorporates uncertainties in the traffic is: (5) as the objective function, which is subjected to the various constraints as in (7), (9), (10), (14), and (15). Also, it must be noted that for $\xi=0$ in (14), the upgraded MILP formulation corresponds to the formulation used for the case of deterministic traffic.

\section{Simulation Results}

The developed MILP is solved for two backbone network topologies shown in Fig. 2 (NSFnet) and Fig. 3 (COST239), with their corresponding traffic demand matrices (Tables I and II) $[1,5]$. To model traffic loads with higher value(s), the base traffic matrix(s) mentioned in the tables are scaled by constant values. Further, we assume that values in traffic matrices correspond to the average traffic for every $s-d$ pair (denoted by $\bar{\Lambda}_{s d}$ ). Also, according to the data in [11], the peak traffic deviation occurs by a value of 2-4 times the average traffic; hence, for our simulation results, we have used the variance-mean ratio $\left(V M R=\left[\tilde{\Lambda}_{s d} / \bar{\Lambda}_{s d}\right]\right)$, and set its values equal to 2,3 , and 4 . The relative cost values for $10 / 40 / 100$ Gbps transponders are set to $1 / 2.5 / 3.75$ respectively $[4,6]$, cost of an amplifier is set to 0.25 per fiber [4], and the cost for electronic processing is fixed to $0.5 x$, where $x=$ bandwidth in Gbps [1]. The modulation format used is Differential Quadrature Phase-Shift Keying (DQPSK), the functionalities and the important components within the various bit-rate transponders are as described in [4], and the dispersion map considered is as specified in [6]. The number of available wavelengths ( $W$ ) is assumed to be 16 wavelengths per link, the type of fiber considered is a standard single-mode fiber (SSMF), and the other parameters used in the study are similar to $[4,6,8,9]$.

For conducting the simulations, we have used the operating system corresponding to Ubuntu and the ILOG CPLEX on a computer with Intel 2 Duo Core having a processor and a memory of $2.2 \mathrm{GHz}$ and $4 \mathrm{~GB}$ respectively, with which, each MILP run for the optimization required approximately 1 to 2 hours.

Fig. 4 shows the overall normalized network cost for a wide range of traffic loads, considering the NSFnet

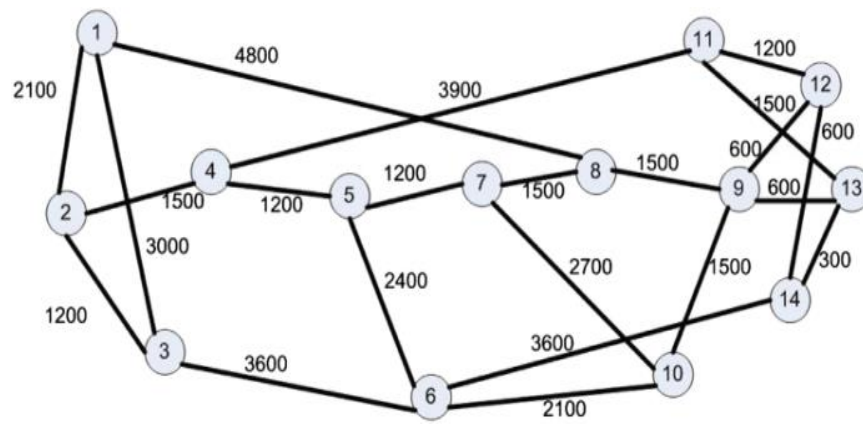

Fig.2. NSFNet backbone topology (with link lengths in $\mathrm{km}$ ) [1].

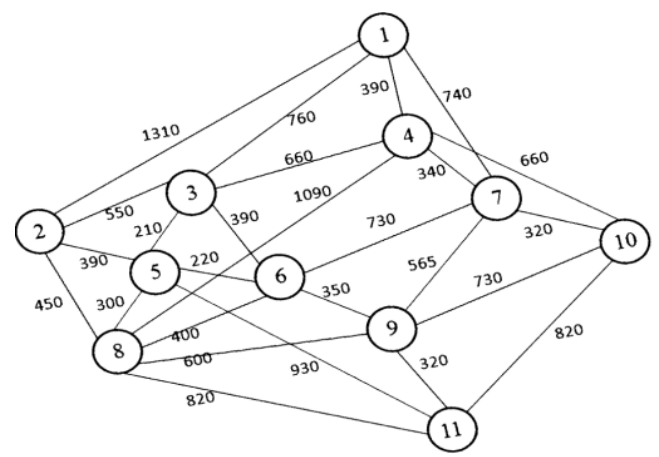

Fig.3. COST239 backbone topology (with link lengths in km) [5].

Table I. The NSFNet backbone network traffic matrix with every entry in Gbps units. (total $=1 \mathrm{Tbps})[1]$.

\begin{tabular}{|c|c|c|c|c|c|c|c|c|c|c|c|c|c|c|}
\hline Node & $\mathbf{1}$ & $\mathbf{2}$ & $\mathbf{3}$ & $\mathbf{4}$ & $\mathbf{5}$ & $\mathbf{6}$ & $\mathbf{7}$ & $\mathbf{8}$ & $\mathbf{9}$ & $\mathbf{1 0}$ & $\mathbf{1 1}$ & $\mathbf{1 2}$ & $\mathbf{1 3}$ & $\mathbf{1 4}$ \\
\hline 1 & 0 & 2 & 1 & 1 & 1 & 4 & 1 & 1 & 2 & 1 & 1 & 1 & 1 & 1 \\
\hline 2 & 2 & 0 & 2 & 1 & 8 & 2 & 1 & 5 & 3 & 5 & 1 & 5 & 1 & 4 \\
\hline 3 & 1 & 2 & 0 & 2 & 3 & 2 & 11 & 20 & 5 & 2 & 1 & 1 & 1 & 2 \\
\hline 4 & 1 & 1 & 2 & 0 & 1 & 1 & 2 & 1 & 2 & 2 & 1 & 2 & 1 & 2 \\
\hline 5 & 1 & 8 & 3 & 1 & 0 & 3 & 3 & 7 & 3 & 3 & 1 & 5 & 2 & 5 \\
\hline 6 & 4 & 2 & 2 & 1 & 3 & 0 & 2 & 1 & 2 & 2 & 1 & 1 & 1 & 2 \\
\hline 7 & 1 & 1 & 11 & 2 & 3 & 2 & 0 & 9 & 4 & 20 & 1 & 8 & 1 & 4 \\
\hline 8 & 1 & 5 & 20 & 1 & 7 & 1 & 9 & 0 & 27 & 7 & 2 & 3 & 2 & 4 \\
\hline 9 & 2 & 3 & 5 & 2 & 3 & 2 & 4 & 27 & 0 & 75 & 2 & 9 & 3 & 1 \\
\hline 10 & 1 & 5 & 2 & 2 & 3 & 2 & 20 & 7 & 75 & 0 & 1 & 1 & 2 & 1 \\
\hline 11 & 1 & 1 & 1 & 1 & 1 & 1 & 1 & 2 & 2 & 1 & 0 & 2 & 1 & 61 \\
\hline 12 & 1 & 5 & 1 & 2 & 5 & 1 & 8 & 3 & 9 & 1 & 2 & 0 & 1 & 81 \\
\hline 13 & 1 & 1 & 1 & 1 & 2 & 1 & 1 & 2 & 3 & 2 & 1 & 1 & 0 & 2 \\
\hline 14 & 1 & 4 & 2 & 2 & 5 & 2 & 4 & 4 & 1 & 1 & 61 & 81 & 2 & 0 \\
\hline
\end{tabular}

Table II. The COST239 backbone network traffic matrix with every entry Gbps units. (total $=1 \mathrm{Tbps})[5]$.

\begin{tabular}{|c|c|c|c|c|c|c|c|c|c|c|c|}
\hline Node & $\mathbf{1}$ & $\mathbf{2}$ & $\mathbf{3}$ & $\mathbf{4}$ & $\mathbf{5}$ & $\mathbf{6}$ & $\mathbf{7}$ & $\mathbf{8}$ & $\mathbf{9}$ & $\mathbf{1 0}$ & $\mathbf{1 1}$ \\
\hline 1 & 0 & 1 & 1 & 3 & 1 & 1 & 1 & 35 & 1 & 1 & 1 \\
\hline 2 & 1 & 0 & 5 & 14 & 40 & 1 & 1 & 10 & 3 & 2 & 3 \\
\hline 3 & 1 & 5 & 0 & 16 & 24 & 1 & 1 & 5 & 3 & 1 & 2 \\
\hline 4 & 3 & 14 & 16 & 0 & 6 & 2 & 2 & 21 & 81 & 9 & 9 \\
\hline 5 & 1 & 40 & 24 & 6 & 0 & 1 & 11 & 6 & 11 & 1 & 2 \\
\hline 6 & 1 & 1 & 1 & 2 & 1 & 0 & 1 & 1 & 1 & 1 & 1 \\
\hline 7 & 1 & 1 & 1 & 2 & 11 & 1 & 0 & 1 & 1 & 1 & 1 \\
\hline 8 & 35 & 10 & 5 & 21 & 6 & 1 & 1 & 0 & 6 & 2 & 5 \\
\hline 9 & 1 & 3 & 3 & 81 & 11 & 1 & 1 & 6 & 0 & 51 & 6 \\
\hline 10 & 1 & 2 & 1 & 9 & 1 & 1 & 1 & 2 & 51 & 0 & 81 \\
\hline 11 & 1 & 3 & 2 & 9 & 2 & 1 & 1 & 5 & 6 & 81 & 0 \\
\hline
\end{tabular}

topology. From the figure it can be seen that with increase in the total traffic, the cost savings decrease for an MLR network due to the requirement of high bit-rate transponders which are required to serve the increasing traffic.

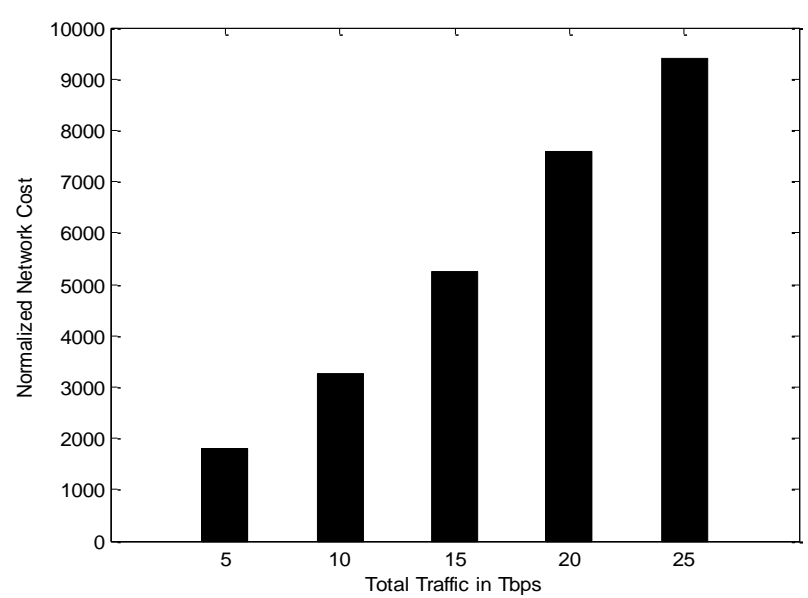

Fig.4. Normalized network cost for MLR in NSFnet. 


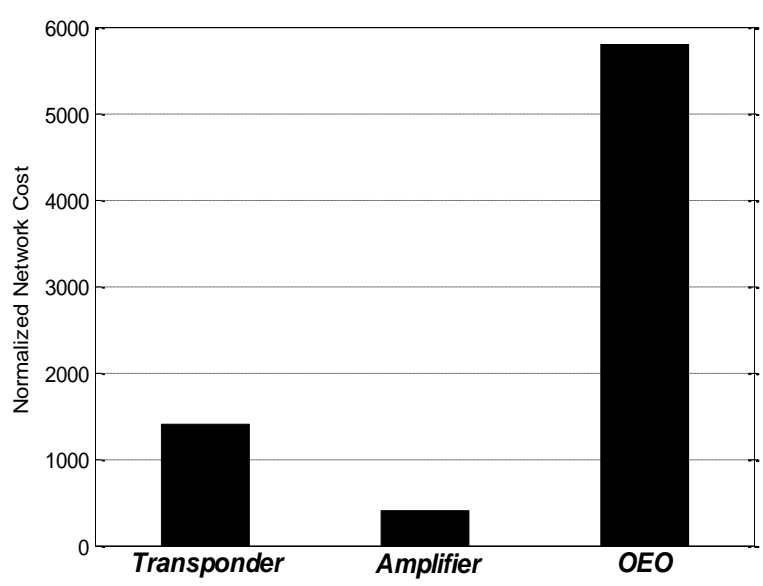

Fig.5. Normalized network cost of different components for MLR in NSFnet.

In Fig. 5, we present the normalized cost for various components for the case of 20 Tbps of total traffic. From the figure it is seen the maximum network cost is incurred owing to the intermediate nodes of the multi-hop lightpath routes requiring $\mathrm{OEO}$ conversion (i.e. electronic processing).

In Fig. 6, considering the NSFnet topology and the average aggregate traffic of $1 \mathrm{Tbps}$, we present the optimized cost-efficient MLR network design as a plot of the normalized cost versus $\xi$, with VMR values of 2, 3, and 4. It can be seen from the figure that as traffic fluctuates from its average value and reaches the maximum (i.e., peak) value of 4 , the network cost increases sharply. Also, it is observed that for small values of $\xi$, the cost increases steadily, and then starts to saturate at a particular higher value of $\xi$. The aforementioned occurs owing to the fact that during the handling of the traffic peaks at the same time in a single or the multiple links of network, the problem of optimization is able to accommodate the 'extra traffic' over the average traffic in an efficient manner. It can also be seen that the network cost increases to an amount where a specific value (in \%) of the links reach the corresponding maximum (or peak) traffic, and thereafter shows almost steady (or constant) behaviour, since, when there occurs a smaller variability (or change) in the statistics of the traffic, such a situation is efficiently handled by the network. Lastly, it can be seen that for lower values of $\xi$, a sudden maximum (or peak) occurs in fewer links since, compared to the deterministic (mean-traffic) case, extra capacity is allocated in such links which is leads to a sharp increase in the cost, which is unlike the situation where a specific capacity is deployed a-priori for handling a specific amount of traffic peaks, with additional peaks in the traffic occurring above them. Thus, based on the network topology, the size of the network, and the distribution of the traffic, there exists a certain value of $\xi$ for which the saturation of cost occurs, and the network also starts to demonstrates robustness to abrupt variations in the traffic. It is observed that in the case of NSFnet, the saturation in cost values start around $\xi=20$.

In Fig. 7, we show the optimized cost-efficient MLR network design for the COST239 network topology. In this case also, the mean total traffic is also set to $1 \mathrm{Tbps}$, and we

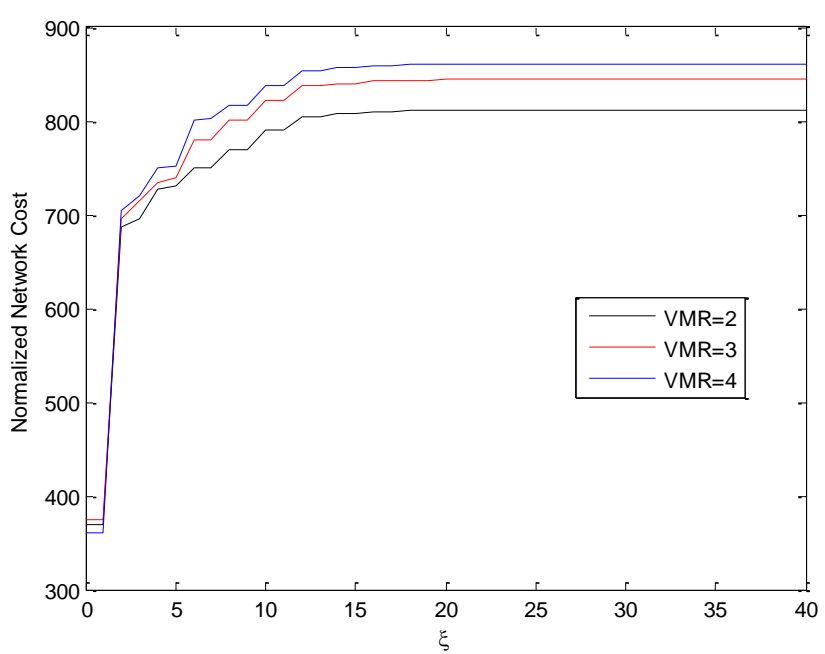

Fig.6. Normalized network cost due to non-deterministic traffic considering the NSFnet topology.

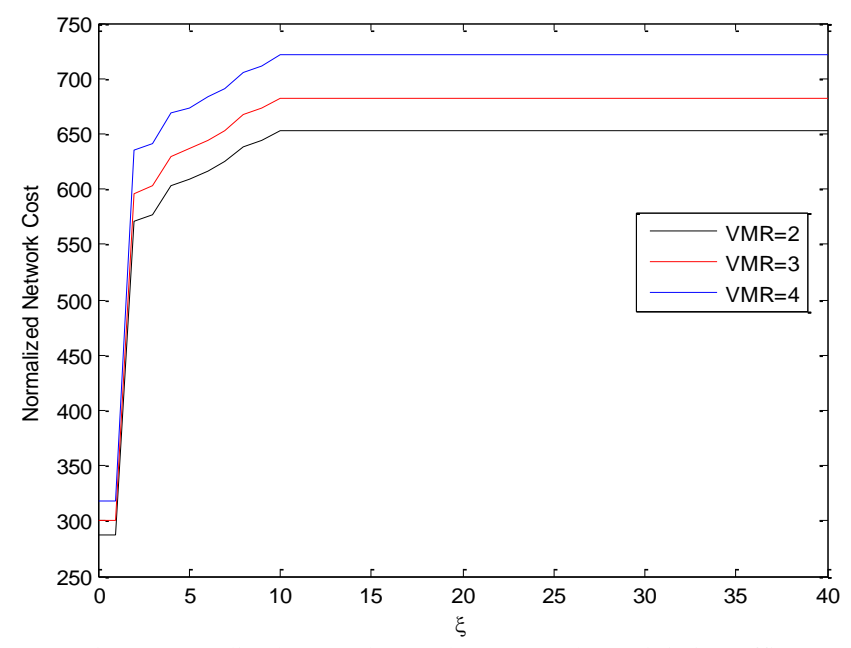

Fig.7. Normalized network cost due to non-deterministic traffic considering the COST 239 topology.

plot the normalized cost versus $\xi$ with various VMR values $(\mathrm{VMR}=2,3$, and 4). It can be seen from the figure that, trend of the normalized network cost versus $\xi$ remains same as in case of the NSFnet topology (Fig. 6). In addition, it can also be seen that, compared to the case of NSFnet for which the saturation in cost values start around $\xi=20$, for the COST239 network topology, the start is around $\xi=10$. From the obtained results, it can be deduced that if the MLR network is optimized in terms of the cost under the assumption that approximately $10-20 \%$ of the demands are at their maximum (peak) value, then the network demonstrates robustness to traffic peaks in approximately all the other demands, and (ii) the saturation of the network cost for a number of source-destination pairs is network topology dependent.

\section{CONCLUSION}

In this study, we proposed a cost-efficient model for the MLR network design which accounts for traffic uncertainties that an actual network may have. For the aforementioned, we employed an optimization technique, and our results show that if the MLR network is cost-optimized under the assumption that approximately $10-20 \%$ of the demands are at their maximum (peak) value, then the robustness to traffic peaks in approximately all the other demands is shown by 
the MLR network. It is also observed that the saturation of network cost for a number of source-destination pairs (i.e., the value of $\xi$ ) is network topology dependent.

Further, the optimization problem is NP-hard, and hence, it is difficult to formulate a heuristic algorithm for the optimization problem considering the uncertainties in the traffic demands. Hence, as a future research problem, we aim to design a heuristic algorithm for a cost-efficient and traffic uncertainty optimized MLR network.

\section{REFERENCES}

[1] A. Nag, M. Tornatore, and B. Mukherjee, "Optical network design with mixed line rates and multiple modulation formats," IEEE $J$. Lightwave Technol., vol. 28, no. 4, pp. 466-475, 2010.

[2] S.P. Singh, S. Sengar, R. Bajpai, and S. Iyer, "Next- Generation Variable-Line-Rate Optical WDM Networks: Issues and Challenges", J. Opt. Commun., De Gruyter, vol. 34, no. 1, pp. 331-350, 2013.

[3] C. Meusburger, D. Schupke, and A. Lord, "Optimizing the migration of channels with higher bitrates," IEEE J. Lightwave Technol., vol. 28, no. 4, pp. 608-615, 2010.

[4] S. Iyer, and S.P. Singh, "Spectral and Power-Efficiency Investigation in Single and Multi-Line-Rate Optical Wavelength Division Multiplexed (WDM) Networks", Photon. Netw. Commun. Springer, vol. 33, no. 1, pp. 39-51, 2017.

[5] P. Chowdhury, M. Tornatore, A. Nag, E. Ip, T. Wang, and B. Mukherjee, "On the design of energy-efficient Mixed-Line-Rate (MLR) optical networks," IEEE J. Lightwave Technol., vol. 30, no. 1, pp. 130-139, 2012
[6] S. Iyer, and S.P. Singh, "Investigation of Launch Power and Regenerator Placement Trade-off in MLR Optical Networks", in Proc. $13^{\text {th }}$ International Conference on Fibre Optics and Photonics (PHOTONICS 2016), OSA Technical Digest (online) (OSA, 2016), Th3A.43, 2016.

[7] A.M.C.A. Koster, M. Kutschka, and C. Raack, "Towards robust network design using integer linear programming techniques," in Proc., Next Generation Internet, NGI, pp. 1-8, 2010.

[8] S. Iyer, and S.P. Singh, "Impact of combined nonlinearities and ASE noise on Performance of 10 Gbps all Optical Star WDM Networks", Communications and Networks, Scientific Research, vol. 3, no. 4, pp 235-249, 2011.

[9] S. Iyer, and S.P. Singh, "Theoretical Evaluation of combined nonlinearities and ASE noise Penalties in Optical Star WDM Networks Based on ITU-T conforming Optical Fibers", IETE Journal of Research, vol. 58, no. 6, pp. 482-492, 2012.

[10] D. Bertsimas and M. Sim, "Robust discrete optimization and network flows," Math. Programming, vol. Ser. B 98, p. 4971, 2003.

[11] "MPLS, OTN, and the rising tide of traffic: The latest turn in the ageold packet versus circuit debate", Juniper Networks, White Paper.Available:http://www.juniper.net/us/en/local/pdf/whitepapers/2 000389-en.pdf 